\title{
SOME PURE RADIATION FIELDS IN GENERAL RELATIVITY
}

\author{
R. P. AKABARI, U. K. DAYE and L. K. PATEL
}

(Received 7 May 1979)

\begin{abstract}
A Demianski-type metric is investigated in connection with Einstein's field equations corresponding to pure radiation fields. With the aid of complex vectorial formalism, a general solution of these field equations is obtained. The solution is algebraically special. A particular case of the solution is considered which includes many known solutions; among them are the radiating versions of some of Kinnersley's solutions.
\end{abstract}

\section{Introduction}

In spite of the fact that an exact gravitational wave solution radiating from a finite source must be algebraically general (Sachs [11]), many investigators have taken a keen interest in obtaining algebraically special solutions of Einstein's field equations. There are several reasons for it. One reason is that the Schwarzschild solution, the Kerr solution [6], the NUT solution (Newman et al. [8]), the Demianski solution [4], and the vacuum solutions of Kinnersley [7] are familiar members of this class.

Many investigators have discussed the non-static generalization of some of the above-mentioned vacuum solutions. Vaidya [12] has obtained a non-static generalization of the Schwarzschild solution, which represents the gravitational field of a spherically symmetric source emitting null fluid. The non-static generalizations of the Kerr and NUT solutions have been treated extensively (see, for example, Vaidya and Patel [14], Vaidya [13] and Vaidya et al. [15]). Patel [9] has obtained the radiating version of Demianski's solution. The object of the present investigation is to obtain radiating versions, which are algebraically special, of some of the vacuum metrics discussed by Kinnersley [7].

The field equations corresponding to the pure radiation fields are

$$
R_{\alpha \beta}=-8 \pi \mu k_{\alpha} k_{\beta}, \quad k_{\alpha} k^{\alpha}=0,
$$

where $\mu$ is the density of flowing radiation. 
The formalism which we are going to use for the derivation of our solutions is the complex vectorial formalism developed by Cahen et al. [1]. A detailed account of this formalism is given by Israel [5], and we shall use his notation. A very brief description of this formalism is given in the next section.

The following conventions are used. The Greek and the first half of the Latin indices will range from 1 to 4 and the second half of the Latin indices will range from 1 to 3 . The Greek indices indicate coordinates and tensor components while the Latin indices indicate tetrad components. The round brackets including the indices will denote symmetrization.

In this paper a radiating space-time will be a simply connected, differentiable, four-dimensional manifold with a metric tensor field $g$ of signature,,,+--that satisfies the field equations (1).

\section{Complex vectorial formalism}

A complete exposition of complex vectorial formalism is not attempted here. We shall consider only those aspects of this formalism which are necessary for our work.

Consider a four-dimensional pseudo-Riemannian space-time manifold $V_{4}$. Let $k_{\alpha}$ and $n_{\alpha}$ be two future pointing real null vector fields and $m_{\alpha}$ be a complex null vector field in $V_{4}$. They are such that the metric on $V_{4}$ has the form

$$
g_{\alpha \beta}=2 k\left({ }_{\alpha} n_{\beta}\right)-2 m\left({ }_{\alpha} \bar{m}_{\beta}\right) \text {, }
$$

with an overhead bar denoting the complex conjugation. Introducing the basic 1-forms,

$$
\theta^{1}=k_{\alpha} d x^{\alpha}, \quad \theta^{2}=m_{\alpha} d x^{\alpha}, \quad \theta^{3}=\overline{\theta^{2}}, \quad \theta^{4}=n_{\alpha} d x^{\alpha} .
$$

Here $x^{\alpha}$ are the local coordinates in $V_{4}$.

Let $Z^{p}$ be a basis for the complex 3-space $\mathscr{C}^{3}$ of self-dual 2-forms, given as

$$
Z^{1}=\theta^{3} \wedge \theta^{4}, \quad Z^{2}=\theta^{1} \wedge \theta^{2}, \quad Z^{3}=\frac{1}{2}\left(\theta^{1} \wedge \theta^{4}-\theta^{2} \wedge \theta^{3}\right),
$$

where $\wedge$ denotes the exterior product.

The metric $\gamma_{p q}$ for the space $\mathscr{C}^{3}$ is given by

$$
\gamma^{p q}=2 \delta_{1}{ }^{(p} \delta_{2}^{q)}-\frac{1}{2} \delta^{p}{ }_{3} \delta^{q}{ }_{3} .
$$

In the absence of torsion in the Riemannian space, the complex-valued connection 1-forms $\sigma_{m}$ and the complex-valued curvature 2-forms $\sum_{p}$ are determined by the following equations known as Cartan's equations of structure:

$$
\left.\begin{array}{l}
d Z^{p}=\frac{1}{2} \varepsilon^{p m n} \sigma_{m} \wedge Z_{n}, \\
\sum_{p}=d \sigma_{p}-\frac{1}{2} \varepsilon_{p m n} \sigma^{m} \wedge \sigma^{n},
\end{array}\right\}
$$


where $d$ denotes exterior differentiation. Since $\sum_{p}$ is a complex 2 -form, it can be expressed in terms of $Z^{p}$ and $\bar{Z}^{p}$ as follows:

$$
\sum_{p}=C_{p q} Z^{q}-\frac{1}{6} R \gamma_{p q} Z^{q}+E_{p \bar{q}} \bar{Z}^{q} \text {. }
$$

Here $C_{p q}$ is a complex-valued trace-free symmetric tensor, which corresponds to the Weyl tensor, $E_{\bar{p} \bar{q}}$ is a hermitian tensor corresponding to the trace-free part of the Ricci tensor and $R$ is the scalar curvature. Note that $C_{p q}$ is related to the five Newman-Penrose components $\psi_{A}$ in terms of which the Petrov classification can be made. In fact,

$$
C_{p q}=2\left(\begin{array}{ccc}
\psi_{0} & -\psi_{2} & \psi_{1} \\
-\psi_{2} & -\psi_{4} & 2 \psi_{3} \\
\psi_{1} & 2 \psi_{3} & -4 \psi_{2}
\end{array}\right)
$$

The Einstein field equations (1) can be expressed in terms of $E_{p \bar{q}}$ as

$$
E_{p \bar{q}}=-8 \pi \mu \delta_{p}^{2} \delta^{\overline{2}} \text {. }
$$

\section{The metric and the field equations}

After obtaining the solutions corresponding to the field equations (9), we intend to obtain the solutions of the Einstein-Maxwell equations corresponding to a source-free electromagnetic field plus pure radiation. So for computational purposes we consider a general metric of the Plebanski-Demianski type [10]. We take the metric in the form

$$
\begin{array}{r}
d s^{2}=2(d u+g G d \beta)(d r+h G d \beta)-2 L(d u+g G d \beta)^{2} \\
-M^{2}\left(\left(d y^{2} /\left(G^{2}\right)\right)+G^{2} d \beta^{2}\right) .
\end{array}
$$

Here $L$ and $M$ are functions of $u, r$ and $y$ and $h, g$ and $G$ are functions of $y$ only. Introducing the basic 1-forms

$$
\left.\begin{array}{rrrr}
\theta^{1} & =d u+g G d \beta, & \sqrt{ } 2 \theta^{2} & =M((d y / G)+i G d \beta), \\
\theta^{3} & =\bar{\theta}^{2}, & \theta^{4} & =d r-L \theta^{1}+h G d \beta,
\end{array}\right\}
$$

we express (10) as

$$
d s^{2}=2\left(\theta^{1} \theta^{4}-\theta^{2} \theta^{3}\right) .
$$

Using the results (4), (6) and (11), we can obtain the connection 1-forms $\sigma_{p}$. They are given by

$$
\left.\begin{array}{rl}
\sigma_{1}= & -2\left[\left(M_{r} / M\right)-i(g G)_{y} /\left(2 M^{2}\right)\right] \theta^{2} \\
\sigma_{2}= & -\sqrt{ } 2\left[i\left(h L_{r} / M+g L_{u} / M\right)+G L_{y} / M\right] \theta^{1} \\
& +2\left[\left(M_{u}+L M_{r}\right) / M+i\left\{(G h)_{y}-L(g G)_{y}\right\} /\left(2 M^{2}\right)\right] \theta^{3}, \\
\sigma_{3}= & -2\left[L_{r}+i\left\{L(g G)_{y}-(G h)_{y}\right\} /\left(2 M^{2}\right)\right] \theta^{1} \\
& -\sqrt{ } 2\left[(M G)_{y} /\left(M^{2}\right)+i\left(g M_{u}+h M_{r}\right) /\left(M^{2}\right)\right] \theta^{2} \\
& -\sqrt{ } 2\left[-(M G)_{y} /\left(M^{2}\right)+i\left(g M_{u}+h M_{r}\right) /\left(M^{2}\right)\right] \theta^{3}+i\left[(g G)_{y} /\left(M^{2}\right)\right] \theta^{4},
\end{array}\right\}
$$


where suffixes denote partial derivatives.

The absence of the terms involving $\theta^{3}$ and $\theta^{4}$ in $\sigma_{1}$ indicates that the null congruence $k^{\alpha}$ is geodesic as well as shear-free.

We can now use $\sigma_{p}$ given by (13) and Cartan's second equation of structure in (6) to determine the curvature 2-forms $\sum_{p}$. The expressions for $\sum_{p}$ are very lengthy and therefore are not given here. These expressions for $\sum_{p}$ will give us $E_{p \bar{q}}$ and $R$. They are given by

$$
\begin{aligned}
& E_{1 \mathrm{~T}}=(2 / M)\left[M_{\mathrm{rr}}-\left\{(g G)_{y}\right\}^{2} /\left(4 M^{3}\right)\right] \text {, } \\
& E_{1 \mathrm{2}}=\bar{E}_{2 \mathrm{I}}=0 \text {, } \\
& E_{1 \mathrm{3}}=\bar{E}_{3 \mathrm{~T}}=(\sqrt{ } 2 / M)\left[h(g G)_{y} M_{r} /\left(M^{3}\right)+g(g G)_{y} M_{u} /\left(M^{3}\right)+G\left(M_{r} / M\right)_{y}\right. \\
& \left.-i\left\{h\left(M_{r} / M\right)_{r}+g\left(M_{r} / M\right)_{u}+G\left((g G)_{y} /\left(2 M^{2}\right)\right)_{y}\right\}\right] \text {, } \\
& E_{2 \overline{2}}=\left(1 / M^{2}\right)\left[h^{2} L_{r r}+2 g h L_{u r}+G^{2} L_{y y}+g^{2} L_{u u}+2 G G_{y} L_{y}\right. \\
& +4 L M M_{\mathrm{ur}}+2 L_{\mathrm{u}} M M_{\mathrm{r}}-2 L_{\mathrm{r}} M M_{\mathrm{u}}+L(g G)_{y}(G h)_{y} /\left(M^{2}\right) \\
& \left.-2\left\{(G h)_{y} /(2 M)\right\}^{2}\right] \text {, } \\
& \bar{E}_{32}=E_{23}=\sqrt{ } 2 L E_{3 \mathrm{~T}}+(\sqrt{ } 2 / M)\left[G\left(L_{r}+M_{u} / M\right)_{y}\right. \\
& +g\left\{2 L(g G)_{y} /\left(2 M^{2}\right)-(G h)_{y} /\left(2 M^{2}\right)\right\}_{u}+i g\left(L_{r}+M_{u} / M\right)_{u} \\
& +h\left\{2 L(g G)_{y} /\left(2 M^{2}\right)-(G h)_{y} /\left(2 M^{2}\right)\right\}_{r}+i h\left(L_{r}+M_{u} / M\right)_{r} \\
& \left.-G\left\{L(g G)_{y} /\left(M^{2}\right)-(G h)_{y} /\left(2 M^{2}\right)\right\}_{y}\right] \text {, } \\
& E_{33}=2\left[L_{r r}-g h M_{u} /\left(M^{3}\right)-h^{2} M_{r} /\left(M^{3}\right)+g^{2} M_{u}{ }^{2} /\left(M^{4}\right)+h^{2} M_{r}{ }^{2} /\left(M^{4}\right)\right. \\
& +\left\{(M G)_{y}\right\}^{2} /\left(M^{4}\right)+2 g h M_{u} M_{r} /\left(M^{4}\right)+2(g G)_{y}(G h)_{y} /\left(M^{4}\right) \\
& -L\left\{(g G)_{y}\right\}^{2} /\left(M^{4}\right)+2 M_{u} M_{r} /\left(M^{2}\right)+2 L M_{r}^{2} /\left(M^{2}\right)-g h M_{r} /\left(M^{3}\right) \\
& \left.-g^{2} M_{u} /\left(M^{3}\right)\right]+\left(2 i /\left(M^{3}\right)\right)\left[M_{r}(G)_{y}+4 L_{r} M(g G)_{y}-M_{u}(g G)_{y}\right. \\
& \left.-L(g G)_{y} M_{r}+\left\{(g G)_{y} /\left(2 M^{2}\right)\right\}_{u} M^{3}\right] \text {, } \\
& R=-E_{3 \overline{3}}+4 L E_{1 \mathrm{~T}}+2 L_{\mathrm{rr}}+4 M_{\mathrm{r}} M_{u} /\left(M^{2}\right)+4\left(M_{\mathrm{r}} / M\right)_{u}+4 L_{r}\left(M_{r} / M\right) \\
& -(G h)_{y}(g G)_{y} /\left(M^{4}\right)+2 L\left\{(g G)_{y}\right\}^{2} /\left(M^{4}\right) \text {. }
\end{aligned}
$$

Now from the field equations (9) we have all $E_{p \bar{q}}=0$ except $E_{2 \overline{2}}$, and $R=0$. Note that $E_{2 \Sigma}$ will give us the radiation density $\mu$.

Equations $E_{1 \mathrm{~T}}=0$ and $E_{1 \overline{3}}=0$ involve only one unknown function $M$. They can be solved to get

where

$$
M^{2}=F^{2}\left(X^{2}+Y^{2}\right), \quad F^{2}=f / Y, \quad 2 f=(g G)_{y},
$$

$$
X_{u}=-(Y-z)_{\theta}, \quad X_{\theta}=(Y-z)_{u}, \quad X_{r}=-1, \quad Y_{r}=0 .
$$

Here, and in what follows, $\theta$ and $z$ are defined by the relations

$$
(g / G) d y=d \theta, \quad(h / G) d y=d z .
$$

We set $R=0$ and use $M^{2}$ given by (15), (16) and (17) to determine the following form of the function $2 L$ :

where

$$
2 L=2 q X+2 J+2\left(E^{*} Y+F^{*} X\right) /\left(X^{2}+Y^{2}\right),
$$

$$
q=-Y_{u} / Y
$$


and $J, E$ and $F$ are functions of $u$ and $y$ subjected to the condition

$$
2 E^{*}+4 J Y-2 X_{u} Y-(h G)_{y} Y / f=0 .
$$

Then considering $E_{3 \overline{3}}=0$ and using the above relations we find that

$$
\begin{array}{r}
2 J=2 X_{u}-Y_{\theta}-(g / F)^{2}\left[\left(Y_{u} /(2 Y)\right)_{u}+\left(Y_{\theta} /(2 Y)\right)_{\theta}-\left(f_{\theta} /(2 f)\right)_{\theta}-f_{\theta} /\left(g^{2}\right)\right. \\
\left.-\left(g G_{\theta}\right)_{\theta} /(G g)\right] .
\end{array}
$$

It then follows from $E_{23}=0$ that

$$
E_{\theta}^{*}=-F_{u}^{*}, \quad E_{u}^{*}=F_{\theta}^{*} .
$$

Using $E_{22}=-8 \pi \mu$, the radiation density $\mu$ can be calculated. The expression for $\mu$ in this general case is lengthy and hence is not given here.

We have, so far, worked with the general metric (10). A case in which $f=Y$ has been treated by Patel [9] in connection with radiating Demianski space-times. In the next section we shall consider one more case which seems to be of physical interest.

\section{The case $f \neq Y, Y=Y(y), G=\sin \alpha$}

We consider the case in which $Y=Y(y)$. It then follows from (16) that

$$
X=a u-r, \quad Y-z=-a \theta+b,
$$

where $a$ and $b$ are constants of integration. No additional constant is added in $X$ because such a constant can always be incorporated in the $r$-coordinate.

Since $Y=Y(y)$ only, equations (20), (21) and (22) imply that

$$
E^{*}=k \theta+w, \quad F^{*}=-k u+m,
$$

where $k, w$ and $m$ are constants of integration. We now introduce a variable $\psi$ and a function $\lambda(\psi)$ as follows:

$$
G=\sin \alpha, \quad(f / Y)^{\frac{1}{2}} d \alpha=d \psi, \quad(f / Y)^{\frac{1}{2}} \sin \alpha=\lambda(\psi) .
$$

Then we find from (21) that

with

$$
2 J=2 a+\frac{\lambda_{\psi \psi}}{\lambda}, \quad E^{*}=-N^{*}-\left[a+\frac{\lambda_{\psi \psi}}{\lambda}\right] Y,
$$

$$
2 N^{*}=(h \sin \alpha)_{\psi} / \lambda .
$$

Now we consider a case in which $\lambda_{\psi \psi} / \lambda$ is a constant, say $\varepsilon$, where $\varepsilon=1,0,-1$. The case $\varepsilon=1$ has been discussed by Patel [9]. Therefore we shall restrict our attention to the following three cases: case (i) $\lambda=e^{\psi}$, case (ii) $\lambda=\sinh _{\psi}$ and case (iii) $\lambda=A \psi+B$, where $A$ and $B$ are constants. 
Here it should be noted that in the above three cases the radiation density $\mu$ is given by

$$
8 \pi \mu=-2 k /\left(X^{2}+Y^{2}\right) .
$$

Now we shall discuss the details of these three cases.

CASE (i). $\lambda=e^{\psi}$.

In this case the results (21)-(24) show that the functions $Y$ and $N^{*}$ satisfy the following differential equations:

and

$$
N_{\psi \psi}^{*}+N_{\psi}^{*}=2(a+1) N^{*}+\{2 a(a+1)+2 k\} Y
$$

$$
Y_{\psi \psi}+Y_{\psi}=-2 N^{*}-2 a Y .
$$

If we set $p^{2}=1+4 k$, then it can be seen easily that the differential equations (29) and (30) are equivalent to the equations

and

$$
Z_{\psi \psi}+Z_{\psi}=(1-p) Z
$$

$$
Z_{\psi \psi}^{*}+Z_{\psi}^{*}=(1+p) Z^{*}
$$

with

and

$$
\left.\begin{array}{l}
Z=N^{*}+\frac{1}{2}(p+2 a+1) Y \\
Z^{*}=N^{*}+\frac{1}{2}(2 a+1-p) Y .
\end{array}\right\}
$$

The solutions of (31) and (32) are given by

and

$$
\left.\begin{array}{l}
Z=C_{1} e^{\frac{1}{2} \psi(-1+\sqrt{ }(5+4 p))}+C_{2} e^{\frac{1}{2} \psi(-1-\sqrt{ }(5+4 p))} \\
Z^{*}=C_{3} e^{-\frac{1}{2} \psi(1-\sqrt{ }(5-4 p))}+C_{4} e^{\frac{1}{2} \psi(-1-\sqrt{ }(5-4 p))}
\end{array}\right\}
$$

where $C_{1}, Z_{2}, C_{3}$ and $C_{4}$ are constants of integration. Knowing $Z$ and $Z^{*}$ from (34) the result (33) gives us $Y$ and $N^{*}$ as

$$
p Y=Z^{*}-Z, \quad 2 p N^{*}=Z(1+2 a-p)+Z^{*}(1+2 a+p) ;
$$

hence we have

and

$$
h \sin \alpha=\int 2 N^{*} e^{\psi} d \psi
$$

$$
\left.g \sin \alpha=-\int 2 Y e^{\psi} d \psi, \quad E^{*}=-N^{*}-(a+1) Y .\right\}
$$

We can therefore obtain the line-element in the final form as

$$
\begin{array}{r}
d s^{2}=2(d u+g \sin \alpha d \beta)(d r+h \sin \alpha d \beta)-2 L(d u+g \sin \alpha d \beta)^{2} \\
-\left(X^{2}+Y^{2}\right)\left(d \psi^{2}+e^{2 \psi} d \beta^{2}\right),
\end{array}
$$


where $X=a u-r, Y, h$ and $g$ are given by (34), (35) and (36) and

$$
2 L=2 a+1+\frac{2 E^{*} Y+2(-k u+m)(a u-r)}{(a u-r)^{2}+Y^{2}},
$$

where $E^{*}$ is given by (36).

Now if we take $k=0$, the radiation density $\mu$ vanishes and we get an empty space-time. We have verified that this empty space-time is the transform of the type $D$ vacuum metric (case II D) of Kinnersley [7]. Thus the metric (37) is the radiative extension of Kinnersley's vacuum metric (case II D). We have also verified that the metric (37) is algebraically special.

CASE (ii). $\lambda=\sinh \psi$.

In this case the differential equations to be satisfied by $Y$ and $N^{*}$ are

and

$$
\left(1-q^{2}\right) Y_{q q}-2 q Y_{q}=2\left(N^{*}+a Y\right)
$$

$$
\left(1-q^{2}\right) N_{q q}^{*}-2 q N_{q}^{*}=2\{-k-a(a+1)\} Y-2(a+1) N^{*},
$$

where $q=\cosh \psi$.

If we set $p^{2}=1+4 k$ and $0<p \leqslant 5 / 4$, then it can be seen easily that the differential equations (39) and (40) are equivalent to the equations

and

$$
\left(1-q^{2}\right) Z_{q q}-2 q Z_{q}+n(n+1) Z=0
$$

$$
\left(1-q^{2}\right) Z_{q q}^{*}-2 q Z_{q}^{*}+l(l+1) Z^{*}=0,
$$

with $1-q=n(n+1), 1+q=l(l+1)$

$$
Z=N^{*}+\frac{1}{2}(1+2 a+p) Y \text { and } Z^{*}=N^{*}+\frac{1}{2}(1+2 a-p) Y .
$$

We need those solutions of (41) and (42) which will give us the Kinnersley vacuum metric (case IIB) for $a=k=0$ as a particular case. The solutions of equations (41) and (42) can be seen from any standard text such as Coddington [3]. They are

$$
Z=a_{1} Q_{n}(q) \text { and } Z^{*}=b_{0} q+b_{1} Q_{\lambda}(q),
$$

where $Q_{n}(q)$ is the Legendre function of the second kind and its series expansion is given by

$$
Q_{n}(q)=1-\frac{n(n+1)}{2 !} q^{2}+\frac{(n+3)(n+1) n(n-2)}{4 !} q^{4}-\ldots
$$

Knowing $Z$ and $Z^{*}$ from (44), the result (43) will give $Y$ and $N^{*}$ as

$$
p Y=Z-Z^{*}, \quad 2 p N^{*}=(p-1-2 a) Z+(p+1+2 a) Z^{*} .
$$

The functions $g$ and $h$ can now be determined as

$$
h \sin \alpha=\int 2 N^{*} \sinh \psi d \psi, \quad g \sin \alpha=-\int 2 Y \sinh \psi d \psi .
$$


Therefore the metric of the solution reduces to

$$
\begin{aligned}
d s^{2}=2 & {\left[d u-\left(\int 2 Y \sinh \psi d \psi\right) d \beta\right]\left[d r+\left(\int 2 N^{*} \sinh \psi d \psi\right) d \beta\right] } \\
& -\left(X^{2}+Y^{2}\right)\left(d \psi^{2}+\sinh ^{2} \psi d \beta^{2}\right)-2 L\left[d \mu-\left(\int 2 Y \sinh \psi d \psi\right) d \beta\right]^{2},
\end{aligned}
$$

where

$$
\begin{aligned}
2 L=1+2 a+\frac{2 E^{*} Y+2(-k u+m) X}{X^{2}+Y^{2}}, \quad X= & a u-r, \\
& \text { and } E^{*}=-N^{*}-(a+1) Y .
\end{aligned}
$$

The functions $Y$ and $N^{*}$ are given by (47). If we put $k=0$, we get $p=1$ and consequently $n=0$ and $l=1$. In this case we get an empty space-time for which $Y$ and $N^{*}$ are given by

and

$$
\left.\begin{array}{l}
Y=a_{1}-b_{0} q-b_{1} Q l(q) \\
N^{*}=-a a_{1}+(a+1)\left[b_{0} q+b_{1} Q_{1}(q)\right] .
\end{array}\right\}
$$

We have verified that for this vacuum solution the Penrose spinors $\psi_{A}$ do not satisfy the equation

$$
2 \psi_{3}^{2}=-3 \psi_{2} \psi_{4} \text {. }
$$

Hence we conclude that this empty space-time is of type II and not of type $D$ (Carmeli and Kaye [2]). However, if $b_{1}=0$, then (51) is satisfied and the metric becomes of type $D$. It is painless to verify that this metric is the transform of Kinnersley's metric (case IIB). Thus the metric (48) represents a radiating Kinnersley's metric (case IIB).

CASE (iii). $\lambda=A \psi+B$.

In this case also we have obtained the following two differential equations for the functions $Y$ and $N^{*}$ :

and

$$
\gamma^{2} Z_{\gamma \gamma}+\gamma Z_{\gamma}+\gamma^{2} Z=0
$$

with

$$
\gamma^{2} Z_{\gamma \gamma}^{*}+\gamma Z_{\gamma}^{*}-\gamma^{2} Z^{*}=0 \text {, }
$$

$$
Z=N^{*}+(a+\sqrt{ } k) \dot{Y}, Z^{*}=N^{*}+(a-\sqrt{ } k) Y \text { and } \gamma=2 \sqrt{ } k(A \psi+B) / A \text {. }
$$

The solutions of (52) and (53) are given by

$$
Z=c_{1} J_{0}(\gamma)+c_{2} K_{0}(\gamma) \text { and } Z^{*}=d_{1} J_{0}(-\gamma)+d_{2} K_{0}(-\dot{\gamma}) \text {, }
$$

where $c_{1}, c_{2}, d_{1}$ and $d_{2}$ are constants and $J_{0}$ and $K_{0}$ are zero-order Bessel functions 
of the first and second kind respectively. In this case we have

$$
\left.\begin{array}{l}
2 \sqrt{ } k Y=Z-Z^{*}, \quad 2 \sqrt{ } k N^{*}=(a+\sqrt{ } k) Z^{*}-(a-\sqrt{ } k) Z, \\
g \sin \alpha=-\int 2 Y(A \psi+B) d \psi, \quad h \sin \alpha=-\int 2 N^{*}(A \psi+B) d \psi,
\end{array}\right\}
$$

where $Z$ and $Z^{*}$ are given by (55).

Here we have assumed that $k$ is positive. The explicit form of the metric in this case can be expressed as

$$
\begin{gathered}
d s^{2}=2\left[d u-\left\{\int 2 Y(A \psi+B) d \psi\right\} d \beta\right]\left[d r+\left\{\int 2 N^{*}(A \psi+B) d \psi\right\} d \beta\right] \\
-\left(X^{2}+Y^{2}\right)\left[d \psi^{2}+(A \psi+B)^{2} d \beta^{2}\right]-2 L\left[d u-\left\{\int 2 Y(A \psi+B) d \psi\right\} d \beta\right]^{2},
\end{gathered}
$$

where

$2 L=2 a+\frac{2 E^{*} Y+2(-k u+m) X}{X^{2}+Y^{2}}, \quad X=a u-r$, and $\quad E^{*}=-N^{*}-a Y$.

The functions $N^{*}$ and $Y$ are given by (56).

Since $Y$ is singular for $k=0$, it follows that the metric (57) is also singular for $k=0$.

\section{References}

[1] M. Cahen, R. Debever and L. Defrise, "A complex vectorial formalism in general relativity", J. Math. and Mech. 16 (1967), 761-786.

[2] M. Carmeli and M. Kaye, "Gravitational field of a rotating body", Ann. Phys. 103 (1977), 97-120.

[3] E. A. Coddington, An introduction to ordinary differential equations (Prentice Hall, New York, 1961), pp. 131-136.

[4] M. Demianski, "New Kerr-line space-time”, Phys. Lett. 42A (1972), 157-159.

[5] W. Israel, "Differential forms in general relativity", Comm. Dublin Inst. for Adv. Studies A 19 (1970), 26-88.

[6] R. P. Kerr, "Gravitational field of a spinning mass as an example of algebraically special metrics", Phys. Rev. Lett. 11 (1963), 237-238.

[7] W. Kinnersley, "Type D vacuum metrics", J. Math. Phys. 10 (1969), 1195-1203.

[8] E. Newman, L. Tamburino and T. Unti, "Empty-space generalization of the Schwartzschild metric", J. Math. Phys. 4 (1963), 915-923.

[9] L. K. Patel, "Radiating Demianski-type space-times", Indian J. Pure Appl. Math. 9 (1978), 1019-1026.

[10] J. F. Plebanski and M. Demianski, "Rotating charged and uniformly accelerating mass in general relativity", Ann. Phys. 98 (1976), 98-127.

[11] R. Sachs, "Gravitational waves in general relativity VI: The out-going radiation condition", Proc. Roy. Soc. A 264 (1961), 309-338. 
[12] P. C. Vaidya, "The gravitational field of a radiating star", Proc. Ind. Acad. Sci. A 33 (1951), 264-279.

[13] P. C. Vaidya, "A generalized Kerr-Schild solution of Einstein's equations", Proc. Camb. Phil. Soc. 75 (1974), 383-390.

[14] P. C. Vaidya and L. K. Patel, "Radiating Kerr metric", Phys. Rev. D 7 (1973), 3590-3593.

[15] P. C. Vaidya, L. K. Patel and P. V. Bhatta, "A Kerr-NUT metric", Gen. Rel. and Grav. 7 (1976), 701-708.

Departments of Mathematics and Statistics

Gujarat University

Ahmedabad-380009

India 